\title{
Coherent states of systems with quadratic Hamiltonians
}

\author{
V.G. Bagrov*, D. M. Gitman`, and A. S. Pereira ${ }^{\ddagger}$
}

October 10, 2018

\begin{abstract}
Different families of generalized CS for one-dimensional systems with general timedependent quadratic Hamiltonian are constructed. In principle, all known CS of systems with quadratic Hamiltonian are members of these families. Some of the constructed generalized CS are close enough to the well-known due to Schrödinger and Glauber CS of a harmonic oscillator, we call them simply CS. However, even among these CS there exist different families of complete sets of CS. These families differ by values of standard deviations at the initial time instant. According to the values of these initial standard deviations one can identify some of the families with semiclassical CS. We discuss properties of the constructed CS, in particular, completeness relations, minimization of uncertainty relations and so on. As a unknown application of the general construction, we consider different CS of an oscillator with a time dependent frequency.

Keywords: Coherent states, quadratic systems
\end{abstract}

\section{Introduction}

\subsection{General}

Coherent states (CS) play an important role in modern quantum theory as states that provide a natural relation between quantum mechanical and classical descriptions. They have a number of useful properties and as a consequence a wide range of applications, e.g. in semiclassical description of quantum systems, in quantization theory, in condensed matter physics, in radiation theory, in quantum computations, in loop quantum gravity, and so on, see, e.g. Refs. [1, 3, 2, 4. Despite the fact that there exist a great number of publications devoted to constructing CS of different systems, an universal definition of CS and a constructive scheme of their constructing for arbitrary physical system is not known. However, it seems that for systems with quadratic Hamiltonians there exist at present a common point of view on this problem1 1 . Starting the works [3, 4, 9, 10, CS are defined as eigenvectors of some annihilation operators that are at the same time integrals of motion, see also 11, 12, 13, 14, 15, 16. Of course such defined CS have to satisfy the corresponding Schrödinger equation. In the frame of such a definition one can in principle construct CS for a general quadratic system. This construction is based on solutions of some classical equations, their analysis represent a nontrivial part of the CS construction.

In this article we, following, the integral of motion method, construct different families of generalized CS for one-dimensional systems with general time-dependent quadratic Hamiltonian. Analyzing these families, we see that some of them are more close to the well-known

\footnotetext{
*Department of Physics, Tomsk State University, Russia; Institute of High Current Electronics, SB RAS, Tomsk, Russia; e-mail: bagrov@phys.tsu.ru

${ }^{\dagger}$ Tomsk State University, Russia; P.N. Lebedev Physical Institute, Russia; Institute of Physics, University of São Paulo, Brazil; e-mail: gitman@if.usp.br

${ }^{\ddagger}$ Institute of Physics, University of São Paulo, Brazil; e-mail: apereira@if.usp.br

${ }^{1}$ In this article we do not discuss the so-called generalized CS [5, 6, 7, 8.
} 
due to Schrödinger and Glauber CS (see [17]) of a harmonic oscillator, we call them simply CS. However, among the latter CS there exist still different families of complete sets of CS. These families differ by values of standard deviations at the initial time instant. According to the values of these initial standard deviations one can identify some of the families with semiclassical CS, as was demonstrated by us in the free particle case [18. We discuss properties of the constructed CS, in particular, completeness relations, minimization of uncertainty relations and so on. As an application of the general construction, we consider CS of an oscillator with a time dependent frequency.

\subsection{Basic equations}

Consider quantum motion of a one-dimensional system with the generalized coordinate $x$ on the whole real axis, $x \in \mathbb{R}=(-\infty, \infty)$, supposing that the corresponding quantum Hamiltonian $\hat{H}_{x}$ is given by a quadratic form of the operator $x$ and the momentum operator $\hat{p}_{x}=-i \hbar \partial_{x}$

$$
\hat{H}_{x}=r_{1} \hat{p}_{x}^{2}+r_{2} x^{2}+r_{3}\left(x \hat{p}_{x}+\hat{p}_{x} x\right)+r_{4} x+r_{5} \hat{p}_{x}+r_{6},
$$

where $r_{s}=r_{s}(t), s=1, \ldots, 6$ are some given functions of the time $t$. We suppose that these functions are real and both $\hat{H}_{x}$ and $\hat{p}_{x}$ are self-adjoint on their natural domains $D_{H_{x}}$ and $D_{p_{x}}$ respectively, see e.g. [19].

Quantum states of the system under consideration are described by a wave function $\Psi(x, t)$ which satisfies the Schrödinger equation

$$
i \hbar \partial_{t} \Psi(x, t)=\hat{H}_{x} \Psi(x, t) .
$$

In what follows, we restrict ourselves by a physically reasonable case $r_{1}(t)>0$. In this case, we introduce dimensionless variables, a coordinate $q$ and a time $\tau$ as follows

$$
q=x l^{-1}, \quad \tau=\int_{0}^{t} \frac{d s}{T(s)}=\frac{2 \hbar}{l^{2}} \int_{0}^{t} r_{1}(s) d s, \quad T(t)=\frac{l^{2}}{2 \hbar r_{1}(t)}
$$

where $l$ is an arbitrary constant of the dimension of the length. The new momentum operator $\hat{p}$ and the new wave function $\psi(q, \tau)$ read

$$
\hat{p}=\frac{l}{\hbar} \hat{p}_{x}=-i \partial_{q}, \quad \psi(q, \tau)=\sqrt{l} \Psi\left(l q, \frac{m l^{2}}{\hbar} \tau\right)
$$

so that $|\Psi(x, t)|^{2} d x=|\psi(q, \tau)|^{2} d q$.

In the new variables, equation (2) takes the form

$$
\hat{S} \psi(q, \tau)=0, \quad \hat{S}=i \partial_{\tau}-\hat{H},
$$

where the new Hamiltonian reads

$$
\hat{H}=\frac{\hat{p}^{2}}{2}+\alpha \hat{q}^{2}+\beta(\hat{q} \hat{p}+\hat{p} \hat{q})+\varrho \hat{q}+\nu \hat{p}+\varepsilon .
$$

Here $\alpha=\alpha(\tau), \beta=\beta(\tau), \varrho=\varrho(\tau), \nu=\nu(\tau)$ and $\varepsilon=\varepsilon(\tau)$,

$$
\begin{aligned}
& \alpha(\tau)=\frac{l^{4}}{2 \hbar^{2}} \frac{r_{2}(t)}{r_{1}(t)}, \beta(\tau)=\frac{l^{2}}{2 \hbar} \frac{r_{3}(t)}{r_{1}(t)}, \varrho(\tau)=\frac{l^{3}}{2 \hbar^{2}} \frac{r_{4}(t)}{r_{1}(t)}, \\
& \nu(\tau)=\frac{l}{2 \hbar} \frac{r_{5}(t)}{r_{1}(t)}, \quad \varepsilon(\tau)=\frac{l^{2}}{2 \hbar^{2}} \frac{r_{6}(t)}{r_{1}(t)},
\end{aligned}
$$

are dimensionless real functions on $\tau$ if $t$ is expressed via $\tau$ by the help of eqs. (3). In what follows, we call $\hat{S}$ the equation operator. 


\section{Constructing time-dependent generalized CS}

\subsection{Integrals of motion linear in canonical operators $\hat{q}$ and $\hat{p}$}

First we construct an integral of motion $\hat{A}(\tau)$ linear in $\hat{q}$ and $\hat{p}$. The general form of such an integral of motion reads

$$
\hat{A}(\tau)=f(\tau) \hat{q}+i g(\tau) \hat{p}+\varphi(\tau),
$$

where $f(\tau), g(\tau)$ and $\varphi(\tau)$ are some complex functions on $\tau$. The operator $\hat{A}(\tau)$ is an integral of motion if it commutes with equation operator (5),

$$
[\hat{S}, \hat{A}(\tau)]=0 .
$$

In the case if the Hamiltonian is self-adjoint, the adjoint operator $\hat{A}^{\dagger}(\tau)$ is also an integral of motion, i.e.,

$$
\left[\hat{S}, \hat{A}^{\dagger}(\tau)\right]=0
$$

The commutator $\left[\hat{A}(\tau), \hat{A}^{\dagger}(\tau)\right]$ reads

$$
\left[\hat{A}(\tau), \hat{A}^{\dagger}(\tau)\right]=\delta=2 \operatorname{Re}\left[g^{*}(\tau) f(\tau)\right] .
$$

Substituting representation (8) into (9), we obtain the following equations for the functions $f(\tau), g(\tau)$, and $\varphi(\tau)$ :

$$
\begin{aligned}
& \dot{f}(\tau)+2 \beta(\tau) f(\tau)-2 i \alpha(\tau) g(\tau)=0, \\
& \dot{g}(\tau)-i f(\tau)-2 \beta(\tau) g(\tau)=0, \\
& \dot{\varphi}(\tau)+\nu(\tau) f(\tau)-i \varrho(\tau) g(\tau)=0 .
\end{aligned}
$$

It is enough to find the functions $f(\tau)$ and $g(\tau)$, then the function $\varphi(\tau)$ can be found by a simple integration. In addition, without loss of the generality we can set $\varphi(0)=0$.

Equations (12) imply that $\delta$ is a real integral of motion, $\delta=$ const. In what follows we suppose that $\delta=1$, which means

$$
\operatorname{Re}\left[g^{*}(\tau) f(\tau)\right]=\operatorname{Re}\left[g^{*}(0) f(0)\right]=1 / 2 .
$$

Any nontrivial solution of two first equations (12) consists of two nonzero functions $f(\tau)$ and $g(\tau)$. That is why we can chose arbitrary integration constants in these equations as

$$
f(0)=c_{1}=\left|c_{1}\right| e^{i \mu_{1}}, \quad g(0)=c_{2}=\left|c_{2}\right| e^{i \mu_{2}}, \quad\left|c_{2}\right| \neq 0, \quad\left|c_{1}\right| \neq 0 .
$$

In terms of the introduced constants, condition (13) yields

$$
\left|c_{2}\right|\left|c_{1}\right| \cos \left(\mu_{1}-\mu_{2}\right)=1 / 2 .
$$

Under the choice $\delta=1$, operators $\hat{A}(\tau)$ and $\hat{A}^{\dagger}(\tau)$ become annihilation and creation operators,

$$
\left[\hat{A}(\tau), \hat{A}^{\dagger}(\tau)\right]=1
$$

It follows from eq. (8) and (13) that

$$
\begin{aligned}
& \hat{q}=g^{*}(\tau)[\hat{A}(\tau)-\varphi(\tau)]+g(\tau)\left[\hat{A}^{\dagger}(\tau)-\varphi^{*}(\tau)\right], \\
& i \hat{p}=f^{*}(\tau)[\hat{A}(\tau)-\varphi(\tau)]-f(\tau)\left[\hat{A}^{\dagger}(\tau)-\varphi^{*}(\tau)\right] .
\end{aligned}
$$


I. We note that the two first equations (12) can be reduced to a one second-order differential equation for the function $g(\tau)$, such an equation has the form of the oscillator equation with a time-dependent frequency $\omega^{2}(\tau)$,

$$
\ddot{g}(\tau)+\omega^{2}(\tau) g(\tau)=0, \quad \omega^{2}(\tau)=2 \alpha-4 \beta^{2}-2 \dot{\beta} .
$$

If we have an exact solution $g(\tau)$ for a given function $\omega^{2}(\tau)$, then the function $f(\tau)$ can be found via the function $g(\tau)$ as

$$
f(\tau)=2 i \beta(\tau) g(\tau)-i \dot{g}(\tau) .
$$

One can chose the functions $\alpha(\tau)$ and $\beta(\tau)$ such that

$$
\omega^{2}(\tau)=2 \alpha(\tau)-4 \beta^{2}(\tau)-2 \dot{\beta}(\tau) .
$$

For example, if we chose

$$
\alpha(\tau)=\frac{1}{2} \omega^{2}(\tau), \beta=\varrho=\nu=\varepsilon=0,
$$

then we are dealing with Hamiltonian of the form

$$
\hat{H}=\frac{\hat{p}^{2}}{2}+\frac{\omega^{2}(\tau)}{2} q^{2} .
$$

II. In addition, the one-dimensional Schrödinger equation

$$
-d_{q}^{2} \Psi(q)+V(q) \Psi(q)=E \Psi(q),
$$

can be identified with eq. (18) if $q \rightarrow \tau, \Psi(q) \rightarrow g(\tau), V(q)-E \rightarrow \omega^{2}(\tau)$.

III. It should be also noted that two first equations (12) can be identified with a particular form of the so-called spin equation, see [20,

$$
i \dot{V}=(\boldsymbol{\sigma} \mathbf{F}) V, \quad V=\left(\begin{array}{c}
f \\
g
\end{array}\right)
$$

with

$$
\mathbf{F}(\tau)=-\frac{1}{2}(2 \alpha+1, i(2 \alpha-1), 4 i \beta)
$$

\subsection{Time-dependent generalized CS}

Let us consider eigenvectors $|z, \tau\rangle$ of the annihilation operator $\hat{A}(\tau)$ corresponding to the eigenvalue $z$,

$$
\hat{A}(\tau)|z, \tau\rangle=z|z, \tau\rangle .
$$

In the general case $z$ is a complex number.

It follows from eqs. (17) and (25) that

$$
\begin{aligned}
& q(\tau) \equiv\langle z, \tau|\hat{q}| z, \tau\rangle=g^{*}(\tau)[z-\varphi(\tau)]+g(\tau)\left[z^{*}-\varphi^{*}(\tau)\right] \\
& i p(\tau) \equiv\langle z, \tau|\hat{p}| z, \tau\rangle=f^{*}(\tau)[z-\varphi(\tau)]-f(\tau)\left[z^{*}-\varphi^{*}(\tau)\right], \\
& z=f(\tau) q(\tau)+i g(\tau) p(\tau)+\varphi(\tau)
\end{aligned}
$$

Using (12), one can easily verify that the functions $q(\tau)$ and $p(\tau)$ satisfy the Hamilton equations

$$
\dot{q}(\tau)=\frac{\partial H}{\partial p}, \quad \dot{p}(\tau)=-\frac{\partial H}{\partial q},
$$


where $H=H(q, p)$ is the classical Hamiltonian that corresponds to the quantum Hamiltonian (6). Thus, the pair $q(\tau)$ and $p(\tau)$ represents a classical trajectory in the phase space of the system under consideration. All such trajectories can be parametrized by the initial data, $q_{0}=q(0)$ and $p_{0}=p(0)$.

Being written in the $q$-representation, eq. (25) reads

$$
\left[f(\tau) q+g(\tau) \partial_{q}+\varphi(\tau)\right]\langle q \mid z, \tau\rangle=z\langle q \mid z, \tau\rangle .
$$

General solution of this equation has the form

$$
\langle q \mid z, \tau\rangle=\Phi_{z}^{c_{1} c_{2}}(q, \tau)=\exp \left[-\frac{f(\tau)}{g(\tau)} \frac{q^{2}}{2}+\frac{z-\varphi(\tau)}{g(\tau)} q+\chi(\tau)\right],
$$

where $\chi(\tau)$ is an arbitrary function on $\tau$.

One can see that the functions $\Phi_{z}^{c_{1} c_{2}}(q, \tau)$ can be written in terms of the mean values $q(\tau)$ and $p(\tau)$ given by eqs. (26),

$$
\Phi_{z}^{c_{1} c_{2}}(q, \tau)=\exp \left\{i p(\tau) q-\frac{f(\tau)}{2 g(\tau)}[q-q(\tau)]^{2}+\tilde{\chi}(\tau)\right\} .
$$

where $\tilde{\chi}(\tau)$ is again an arbitrary function on $\tau$.

The functions $\Phi_{z}^{c_{1} c_{2}}$ satisfy the following equation

$$
\hat{S} \Phi_{z}^{c_{1} c_{2}}(q, \tau)=\lambda(\tau) \Phi_{z}^{c_{1} c_{2}}(q, \tau),
$$

where

$$
\lambda(\tau)=i \partial_{\tau} \tilde{\chi}(\tau)+\alpha q^{2}(\tau)-\frac{1}{2}\left[p^{2}(\tau)+\frac{f}{g}\right]-i \nu p(\tau)-\beta-i \varepsilon .
$$

If we wish the functions (29) satisfies the Schrödinger equation (5), we have to fix $\tilde{\chi}(\tau)$ from the condition $\lambda(\tau)=0$. Thus, we obtain for the function $\tilde{\chi}(\tau)$ the following result:

$$
\begin{aligned}
& \tilde{\chi}(\tau)=\phi(\tau)+\ln N \\
& \phi(\tau)=\int_{0}^{\tau}\left\{i \alpha q^{2}(\tau)-\frac{i}{2}\left[p^{2}(\tau)+\frac{f}{g}\right]-i \nu p(\tau)-\beta-i \varepsilon\right\} d \tau,
\end{aligned}
$$

were $N$ is a normalization constant, which we suppose to be real.

The probability densities generated by the wave functions (29) have the form

$$
\rho_{z}^{c_{1} c_{2}}(q, \tau)=\left|\Phi_{z}^{c_{1} c_{2}}(q, \tau)\right|^{2}=N^{2} \exp \left\{-\frac{[q-q(\tau)]^{2}}{2|g(\tau)|^{2}}+2 \operatorname{Re} \phi(\tau)\right\} .
$$

Considering the normalization integral, we find the constant $N$,

$$
\int_{-\infty}^{\infty} \rho_{z}^{c_{1} c_{2}}(q, \tau) d q=1 \Rightarrow N=\frac{\exp (-\operatorname{Re} \phi(\tau))}{\sqrt{\sqrt{2 \pi}|g(\tau)|}} .
$$

Thus, normalized solutions of the Schrödinger equation that at the same time are eigenfunctions of the annihilation operator $\hat{A}(\tau)$ have the form

$$
\Phi_{z}^{c_{1} c_{2}}(q, \tau)=\frac{1}{\sqrt{\sqrt{2 \pi}|g(\tau)|}} \exp \left\{i p(\tau) q-\frac{f(\tau)}{g(\tau)} \frac{[q-q(\tau)]^{2}}{2}+i \operatorname{Im} \phi(\tau)\right\}
$$

and the corresponding probability densities read

$$
\rho_{z}^{c_{1} c_{2}}(q, \tau)=\frac{1}{\sqrt{2 \pi}|g(\tau)|} \exp \left\{-\frac{[q-q(\tau)]^{2}}{2|g(\tau)|^{2}}\right\} .
$$

In what follows we call the solutions (35) the time-dependent generalized CS. 


\section{Time-dependent CS of quadratic systems}

Using eqs. (17) and (25) we can calculate standard deviations $\sigma_{q}(\tau), \sigma_{p}(\tau)$, and the quantity $\sigma_{q p}(\tau)$, in the generalized CS,

$$
\begin{aligned}
& \sigma_{q}(\tau)=\sqrt{\left\langle(\hat{q}-\langle q\rangle)^{2}\right\rangle}=\sqrt{\left\langle q^{2}\right\rangle-\langle q\rangle^{2}}=|g(\tau)|, \\
& \sigma_{p}(\tau)=\sqrt{\left\langle(\hat{p}-\langle p\rangle)^{2}\right\rangle}=\sqrt{\left\langle p^{2}\right\rangle-\langle p\rangle^{2}}=|f(\tau)|, \\
& \sigma_{q p}(\tau)=\frac{1}{2}\langle(\hat{q}-\langle q\rangle)(\hat{p}-\langle p\rangle)+(\hat{p}-\langle p\rangle)(\hat{q}-\langle q\rangle)\rangle \\
& =i\left[1 / 2-g(\tau) f^{*}(\tau)\right] .
\end{aligned}
$$

One can easily see that the generalized CS (35) minimize the Robertson-Schrödinger uncertainty relation [21,

$$
\sigma_{q}^{2}(\tau) \sigma_{p}^{2}-\sigma_{q p}^{2}(\tau)=1 / 4
$$

This means that the generalized CS are squeezed states [4.

Let us analyze the Heisenberg uncertainty relation in the generalized CS taking into account restriction (13),

$$
\left.\sigma_{q}(\tau) \sigma_{p}(\tau)\right|_{2 \operatorname{Re}\left(c_{1}^{*} c_{2}\right)}=\frac{1}{2} \sqrt{1+4\left(\operatorname{Im}\left(g f^{*}\right)\right)^{2}} \geq \frac{1}{2} .
$$

Then using (37), we find $\sigma_{q}(0)=\sigma_{q}=\left|c_{2}\right|$ and $\sigma_{p}(0)=\sigma_{p}=\left|c_{1}\right|$, such that at $\tau=0$ this relation reads

$$
\left.\sigma_{q} \sigma_{p}\right|_{2 \operatorname{Re}\left(c_{1}^{*} c_{2}\right)}=\sqrt{\frac{1}{4}+\left[\left|c_{2}\right|\left|c_{1}\right| \sin \left(\mu_{2}-\mu_{1}\right)\right]^{2}} .
$$

Taking into account eqs. (14), we see that if $\mu_{1}=\mu_{2}=\mu$ the left hand side of (39) is minimal, such that

$$
\sigma_{q} \sigma_{p}=1 / 2, \quad \sigma_{q p}=0 .
$$

One can see that the constant $\mu$ does not enter CS (35). Then, in what follows we consider generalized CS with the restriction $\mu_{1}=\mu_{2}=\mu=0$. Namely, such states we call simply CS.

Now restriction (13) takes the form $c_{1}=\left|c_{1}\right|, c_{2}=\left|c_{2}\right|, 2 c_{1}=c_{2}^{-1}$, such that

$$
g(0)=\left|c_{2}\right|=\sigma_{q}, \quad f(0)=\left|c_{1}\right|=\sigma_{p}=\frac{1}{2 \sigma_{q}} .
$$

Thus, $\sigma_{q p}=\sigma_{q p}(0)=i[1 / 2-g(0) f(0)]=0$, which is consistent with eqs. (41).

With account taken of eqs. (35), (37) and (42), we obtain the following expression for the CS:

$$
\begin{aligned}
& \Phi_{z}^{\sigma_{q}}(q, \tau)=\frac{1}{\sqrt{\sqrt{2 \pi} \sigma_{q}(\tau)}} \exp \left\{i p(\tau) q-\frac{f(\tau)}{g(\tau)} \frac{[q-q(\tau)]^{2}}{2}+i \operatorname{Im} \phi(\tau)\right\}, \\
& \phi(\tau)=\int_{0}^{\tau}\left\{i \alpha q^{2}(\tau)-\frac{i}{2}\left[p^{2}(\tau)+\frac{f(\tau)}{g(\tau)}\right]-i \nu p(\tau)-\beta-i \varepsilon\right\} d \tau .
\end{aligned}
$$

In fact, we have a family of CS parametrized by one real parameter-the initial standard deviation $\sigma_{q}>0$. Each set of CS in the family has its specific initial standard deviations $\sigma_{q}$. Different CS from a family with a given $\sigma_{q}$ have different quantum numbers $z$, which 
are in one to one correspondence with trajectory initial data $q_{0}$ and $p_{0}$. It follows from eq. (26) that

$$
z=\frac{q_{0}}{2 \sigma_{q}}+i \sigma_{q} p_{0}, \quad q_{0}=2 \sigma_{q} \operatorname{Re} z, \quad p_{0}=\frac{\operatorname{Im} z}{\sigma_{q}} .
$$

The probability density that corresponds to the CS (43) reads

$$
\rho_{z}^{\sigma_{q}}(q, \tau)=\frac{1}{\sqrt{2 \pi} \sigma_{q}(\tau)} \exp \left\{-\frac{[q-q(\tau)]^{2}}{2 \sigma_{q}^{2}(\tau)}\right\}
$$

One can prove that for any fixed $\sigma_{q}$ states (43) form an over complete set of functions with the following orthogonality and completeness relations

$$
\begin{aligned}
& \int \overline{\Phi_{z^{\prime}}^{\sigma_{q}}(q, \tau)} \Phi_{z}^{\sigma_{q}}(q, \tau) d q=\exp \left(z^{*} z-\frac{\left|z^{\prime}\right|^{2}+|z|^{2}}{2}\right), \quad \forall \tau \\
& \iint \Phi_{z}^{\sigma_{q}}(q, \tau) \overline{\Phi_{z}^{\sigma_{q}}\left(q^{\prime}, \tau\right)} d^{2} z=\pi \delta\left(q-q^{\prime}\right), \quad d^{2} z=d \operatorname{Re} z d \operatorname{Im} z, \quad \forall \tau .
\end{aligned}
$$

\section{An exact solution of oscillator equation with time- dependent frequency and related CS}

Let us consider the following function $\omega^{2}(\tau)$,

$$
\omega^{2}(\tau)=\omega^{2}+\frac{2 \omega_{0}^{2}}{\cosh ^{2} \omega_{0} \tau}, \quad \omega^{2} \leq \omega^{2}(\tau) \leq \omega_{\max }^{2}, \quad \omega( \pm \infty)=\omega^{2},
$$

where $\omega$ and $\omega_{0}$ are some positive constants, $\omega_{\max } \geq \omega$. The function $\omega^{2}(\tau)$ is an even function, which decreases monotonically as $|\tau|$ changes from 0 to $\infty$,

$$
\omega^{2}( \pm \infty)=\omega^{2}, \omega^{2}(0)=\omega_{\max }^{2}=\omega^{2}+2 \omega_{0}^{2} .
$$

For $\omega=1$ and $\omega_{0}=2^{-1 / 2}$, the plot of the function $\omega(\tau)$ has the form

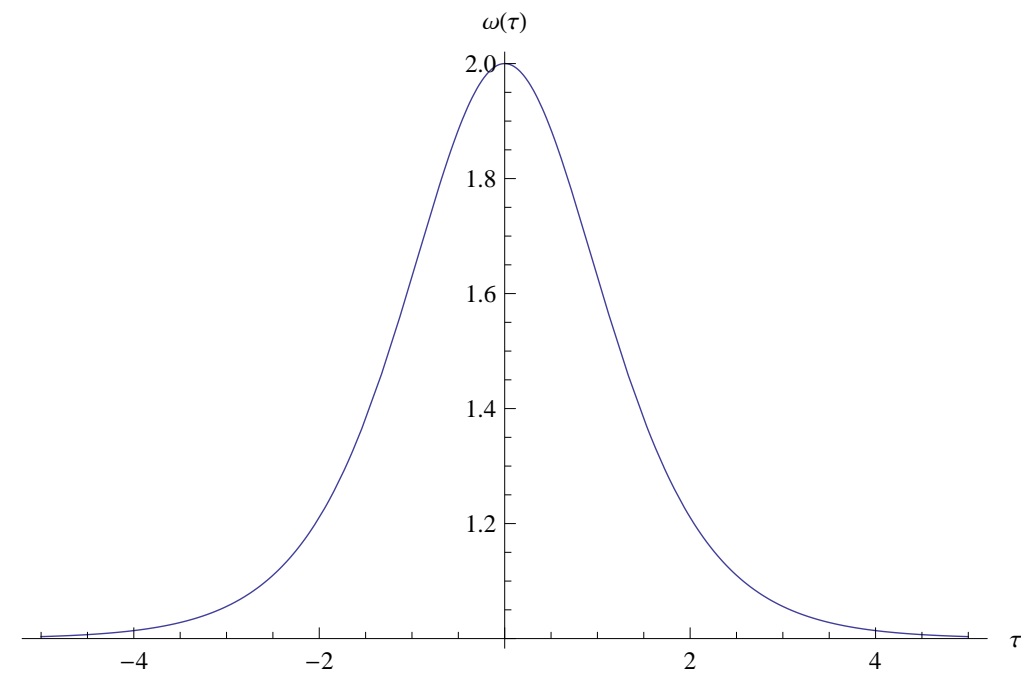

The general solution of equation (18) can be written as

$$
g(\tau)=\left[\frac{i A \omega_{0} \tanh \left(\omega_{0} \tau\right)}{\omega^{2}+\omega_{0}^{2}}+B\right] \cos (\omega \tau)+\left[\frac{i A \omega^{2}}{\omega^{2}+\omega_{0}^{2}}-B \omega_{0} \tanh \left(\omega_{0} \tau\right)\right] \frac{\sin (\omega \tau)}{\omega}
$$


The restriction (13), (14), and (42) that set the CS from the entire set of generalized CS lead to the following relations for the constants $A$ and $B$ :

$$
B=g(0)=|g(0)|=\sigma_{q}, \quad A=f(0)=|f(0)|=1 / 2 \sigma_{q} .
$$

Using eqs. (49) and (50) we calculate the mean trajectories $q(\tau)$ and $p(\tau)$ according eqs. (26)

$$
\begin{aligned}
& q(\tau)=2 \operatorname{Re}\left[g(\tau) z^{*}\right]=\left.g(\tau)\right|_{A=\bar{A}, B=\bar{B}}, \\
& p(\tau)=\left.\dot{g}(\tau)\right|_{A=\bar{A}, B=\bar{B}}=\dot{q}(\tau), \bar{A}=-\sigma_{q}^{-1} \operatorname{Im} z, \bar{B}=2 \sigma_{q} \operatorname{Re} z .
\end{aligned}
$$

For $\omega_{\min }>0$ the mean trajectory $q(\tau)$ can be presented as

$$
q(\tau)=R_{0} R(\tau) \sin \left[\omega \tau+\Theta(\tau)+\Theta_{0}\right],
$$

where functions $R(\tau)$ and $\Theta(\tau)$ and constants $R_{0}$ and $\Theta_{0}$ are

$$
\begin{aligned}
& R(\tau)=\sqrt{1+\frac{\omega_{0}^{2}}{\omega^{2}} \tanh ^{2} \omega_{0} \tau}, \quad 1 \leqslant R(\tau)<\omega^{-1} \sqrt{\omega^{2}+\omega_{0}^{2}} ; \\
& \Theta(\tau)=\arctan \left[\frac{\omega_{0}}{\omega} \tanh \omega_{0} \tau\right], \quad-\Delta<\Theta(\tau)<\Delta, \quad \Delta=\arctan \left(\frac{\omega_{0}}{\omega}\right) ; \\
& R_{0}=\frac{\sqrt{p_{0}^{2} \omega^{2}+q_{0}^{2}\left(\omega^{2}+\omega_{0}^{2}\right)^{4}}}{\left(\omega^{2}+\omega_{0}^{2}\right)^{2}}, \quad \sin \Theta_{0}=\frac{q_{0}}{R_{0}}, \quad \cos \Theta_{0}=\frac{p_{0} \omega}{R_{0}\left(\omega^{2}+\omega_{0}^{2}\right)^{2}} .
\end{aligned}
$$

Thus, we deal with a quasiharmonic motion with the frequency $\omega$ and an amplitude that is changing in time in finite limits and with a time-dependent phase that is slowly changing in also finite limits.

Let us derive the case of a harmonic oscillator with a fixed frequency $\omega>0$ from the above formulas. To this end we have to set $\omega_{0}=0$ and $\alpha=\omega^{2} / 2, \nu=\beta=\varepsilon=0$ such that $\omega^{2}(\tau)=\omega^{2}$. Then

$$
\begin{aligned}
& g(\tau)=\sigma_{q} \cos \omega \tau+\frac{i \sin \omega \tau}{2 \sigma_{q} \omega}, \quad f(\tau)=\frac{\cos \omega \tau}{2 \sigma_{q}}+i \sigma_{q} \omega \sin \omega \tau \\
& q(\tau)=q_{0} \cos \omega \tau+\frac{p_{0}}{\omega} \sin \omega \tau, p(\tau)=p_{0} \cos \omega \tau-\omega q_{0} \sin \omega \tau
\end{aligned}
$$

and $z=\sigma_{p} q_{0}+i \sigma_{q} p_{0}$. Taking all that into account in Eq. (43), we obtain the following representation for CS (in the above given definition) of the harmonic oscillator:

$$
\Phi_{z}^{\sigma_{q}}(q, \tau)=\frac{1}{\sqrt{\sqrt{2 \pi} g(\tau)}} \exp \left\{-\frac{1}{2} \frac{f(\tau)}{g(\tau)}\left[q-\frac{z}{f(\tau)}\right]^{2}+\frac{f^{*}(\tau)}{f(\tau)} \frac{z^{2}}{2}-\frac{|z|^{2}}{2}\right\} .
$$

For these CS

$$
\sigma_{q}(\tau)=\sigma_{q} \sqrt{1+\frac{\left(1-4 \sigma_{q}^{4} \omega^{2}\right)}{4 \sigma_{q}^{4} \omega^{2}} \sin ^{2} \omega \tau}, \quad \sigma_{p}(\tau)=\sigma_{p} \sqrt{1-\left(1-4 \sigma_{q}^{4} \omega^{2}\right) \sin ^{2} \omega \tau},
$$

and the corresponding probability density reads

$$
\rho_{z}^{\sigma_{q}}(q, \tau)=\frac{1}{\sqrt{2 \pi} \sigma_{q}(\tau)} \exp \left\{-\frac{[q-q(\tau)]^{2}}{2 \sigma_{q}^{2}(\tau)}\right\} .
$$

One has to consider the following three cases: 
a) $\sigma_{q} \sqrt{2 \omega}=1$, then

$$
\sigma_{q}(\tau)=\sigma_{q}, \quad \sigma_{p}(\tau)=\sigma_{p}, \quad \sigma_{q}(\tau) \sigma_{p}(\tau)=\sigma_{q} \sigma_{p}=1 / 2, \forall \tau .
$$

b) $\sigma_{q} \sqrt{2 \omega}<1$, then

$$
\begin{aligned}
& \left.\sigma_{q}(\tau)\right|_{\min }=\left.\sigma_{q}(\tau)\right|_{\tau=\frac{\pi n}{\omega}}=\sigma_{q},\left.\quad \sigma_{q}(\tau)\right|_{\max }=\left.\sigma_{q}(\tau)\right|_{\tau=\frac{2 n+1}{2} \frac{\pi}{\omega}}=\frac{1}{2 \sigma_{q} \omega}, \\
& \left.\sigma_{p}(\tau)\right|_{\min }=\left.\sigma_{p}(\tau)\right|_{\tau=\frac{2 n+1}{2} \frac{\pi}{\omega}}=\sigma_{q} \omega,\left.\quad \sigma_{p}(\tau)\right|_{\max }=\left.\sigma_{p}(\tau)\right|_{\tau=\frac{\pi n}{\omega}}=\sigma_{p}, \\
& \left.\sigma_{q}(\tau) \sigma_{p}(\tau)\right|_{\min }=\left.\sigma_{q}(\tau) \sigma_{p}(\tau)\right|_{\tau=\frac{\pi n}{\omega}}=1 / 2, \\
& \left.\sigma_{q}(\tau) \sigma_{q}(\tau)\right|_{\max }=\left.\sigma_{q}(\tau) \sigma_{q}(\tau)\right|_{\tau=\frac{2 n+1}{4} \frac{\pi}{\omega}}=\frac{1+4 \sigma_{q}^{4} \omega^{2}}{8 \sigma_{q}^{2} \omega}, \quad n \in \mathbb{N}=0,1,2, \ldots .
\end{aligned}
$$

c) $\sigma_{q} \sqrt{2 \omega}>1$, then

$$
\begin{aligned}
& \left.\sigma_{q}(\tau)\right|_{\min }=\left.\sigma_{q}(\tau)\right|_{\tau=\frac{2 n+1}{2} \frac{\pi}{\omega}}=\frac{1}{2 \sigma_{q} \omega},\left.\quad \sigma_{q}(\tau)\right|_{\max }=\left.\sigma_{q}(\tau)\right|_{\tau=\frac{n \pi}{\omega}}=\sigma_{q}, \\
& \left.\sigma_{p}(\tau)\right|_{\min }=\left.\sigma_{p}(\tau)\right|_{\tau=\frac{n \pi}{\omega}}=\sigma_{p},\left.\quad \sigma_{p}(\tau)\right|_{\max }=\left.\sigma_{p}(\tau)\right|_{\tau=\frac{2 n+1}{2} \frac{\pi}{\omega}}=\sigma_{q} \omega, \\
& \left.\sigma_{q}(\tau) \sigma_{p}(\tau)\right|_{\min }=\left.\sigma_{q}(\tau) \sigma_{p}(\tau)\right|_{\tau=\frac{n}{2} \frac{\pi}{\omega}}=1 / 2, \\
& \left.\sigma_{q}(\tau) \sigma_{q}(\tau)\right|_{\max }=\left.\sigma_{q}(\tau) \sigma_{q}(\tau)\right|_{\tau=\frac{2 n+1}{4} \frac{\pi}{\omega}}=\frac{1+4 \sigma_{q}^{4} \omega^{2}}{8 \sigma_{q}^{2} \omega}, \quad n \in \mathbb{N} .
\end{aligned}
$$

We can see that in the case a) the Heisenberg uncertainty relation is minimized in the CS (55). In the same case these CS coincide (up to a phase factor) with the wellknown Schrödinger-Glauber CS 17. CS with $\sigma_{q}$ obeying b) and c) minimize the Heisenberg uncertainty relation periodically, but the product $\sigma_{q}(\tau) \sigma_{q}(\tau)$ is always restricted by the limits $1 / 2$ and $\frac{1+4 \sigma_{q}^{4} \omega^{2}}{8 \sigma_{q}^{2} \omega}$.

Setting $\omega_{0}=\omega=\alpha=\nu=\beta=\varepsilon=0$, and taking into account the limits

$$
\begin{aligned}
& \lim _{\omega_{0} \rightarrow 0} g(\tau)=B \cos (\omega \tau)+\frac{i A}{\omega} \sin (\omega \tau), \lim _{\omega_{0}, \omega \rightarrow 0} g(\tau)=B+i A \tau, \\
& \lim _{\omega \rightarrow 0} g(\tau)=\left(i A-B \omega_{0}^{2} \tau\right) \frac{\tanh \left(\omega_{0} \tau\right)}{\omega_{0}}+B,
\end{aligned}
$$

we obtain from (43) CS of a free particle studied by us in the Ref. [18].

\section{Acknowledgements}

Bagrov thanks FAPESP for support and IF USP for hospitality;

Gitman thanks CNPq and FAPESP for permanent support;

The work of Bagrov and Gitman is also partially supported by Tomsk State University A. S. Competitiveness Improvement Program;

Pereira thanks FAPESP for support.

\section{References}

[1] Klauder J R, Sudarshan E C, Fundamentals of Quantum Optics, (Benjamin, 1968); Klauder I R, Skagerstam B S, Coherent States, Applications in Physics and Mathematical Physics, (World Scientific, Singapore, 1985); Perelomov A M, Generalized Coherent 
States and Their Applications, (Springer-Verlag, 1986); Gazeau J P, Coherent States in Quantum Physics, (Wiley-VCH, Berlin, 2009); Nielsen M, Chuang I, Quantum Computation and Quantum Information (Cambridge University Press, Cambridge, England, 2000)

[2] Malkin I A and Man'ko V I 1968 Zh. Eksp. Teor. Fiz. 551014

[3] Malkin I A, Man'ko V I, Dynamical Symmetries and Coherent States of Quantum Systems, (Nauka, Moscow, 1979)

[4] V.V. Dodonov, V.I. Man'ko, Invariants and correlated states of nonstationary quantum systems. In: Invariants and the Evolution of Nonstationary Quantum Systems. Proceedings of Lebedev Physics Institute, 183,M.A. Markov, ed. (Nauka, Moscow 1987) 71-181, [translated by Nova Science, Commack, New York, 1989, pp. 103-261

[5] R. Gilmore, Geometry of symmetrized states, Ann. Phys. (NY) 74 391-463 (1972).

[6] A. M. Perelomov, Coherent states for arbitrary Lie groups, Commun. Math. Phys. 26 222-236 (1972).

[7] A.M.Perelomov, Generalized Coherent States and Their Applications, Springer-Verlag, 1986.

[8] S.T. Ali, J-P. Antoine, and J-P. Gazeau, Coherent States, Wavelets and Their Generalizations, Springer-Verlag, New York, Berlin, Heidelberg, 2000.

[9] Theory of Nonclassical States of Light, Edited by V. V. Dodonov and V. I. Man'ko (Taylor \& Francis Group, London, NY 2003)

[10] V.G. Bagrov, I.L. Buchbinder and D.M. Gitman, Coherent states of a relativistic particle in an external electromagnetic field, Journ. Phys. A 9 (1976) 1955-1965

[11] J.P. Gazeau, M.C. Baldiotti, and D.M. Gitman, Semiclassical and quantum motion on non-commutative plane, Physics Letters A, 373, (43) (2009) 3937-3943

[12] V.G. Bagrov, S.P. Gavrilov, D.M. Gitman, and D. P. Meira Filho, Coherent states of non-relativistic electron in magnetic-solenoid field, Journ. Physics. A43 (2010) 3540169 (10 pages); Coherent and semiclassical states in magnetic field in the presence of the Aharonov-Bohm solenoid, J. Phys. A: Math. Theor. 44 (2011) 055301

[13] V.G. Bagrov, S.P. Gavrilov, D.M. Gitman, and K. Gorska, Completeness for coherent states in magnetic-solenoid field, Journ. Phys. A 45 (2012) 244008 (11pp)

[14] V.G. Bagrov, J.-P. Gazeau, D. Gitman, and A. Levine, Coherent states and related quantizations for unbounded motions, Journ. Phys. A 45 (2012) 125306

[15] V.G. Bagrov, D. M. Gitman, E. S. Macedo, and A. S. Pereira, Coherent states of inverse oscillator and related problems, Journ. Phys. A: Math. Theor. 46 (2013) 325305 (13pp);

[16] V.G. Bagrov, D.M. Gitman, A. Pereira, Coherent and semiclassical states of a free particle, Uspekhi Fizicheskikh Nauk 184 (9) (2014) 961-966, Physics-Uspekhi 57 (9) (2014) 891-896

[17] E. Schrödinger, Der stetige Übergang von der Mikro- zur Makro-mechanik, Naturwissenschaften, Bd. 14, H. 28 (1926) 664-666; R.J. Glauber, The quantum theory of optical coherence, Phys. Rev. 130 No. 6 (1963) 2529-2539; Coherent and incoherent states of the radiation field, Phys. Rev. 131 No. 6 (1963) 2766-2788 
[18] V.G. Bagrov, D.M. Gitman, A. Pereira, Coherent and semiclassical states of a free particle, Physics-Uspekhi 57 (9) (2014) 891-896

[19] Bagrov V G, Gitman D M, Exact Solutions of Relativistic Wave Equations (Kluwer Acad. Publisher, Boston 1990); Dirac Equation and its Solutions (de Gruyter, Boston, 2014) pp. 444

[20] V.G. Bagrov, M.C. Baldiotti, D.M. Gitman, A.D. Levin, Spin equation and its solutions, Ann. der Physik 14 [11-12] (2005) 764-789

[21] Schrödinger E, Sitzungsberichte Preus. Acad. Wiss., Phys.-Math. Klasse, 19296 (Berlin 1930) ; Robertson H P, Phys. Rev. 35667 (1930) 